\title{
The EUV spectrum of the Sun: Quiet- and active-Sun irradiances and chemical composition ${ }^{\star}$
}

\author{
G. Del Zanna
}

\begin{abstract}
DAMTP, Centre for Mathematical Sciences, University of Cambridge, Wilberforce Road, Cambridge CB3 0WA, UK
e-mail: g.del-zanna@damtp.cam.ac.uk
\end{abstract}

Received 12 December 2018 / Accepted 28 January 2019

\begin{abstract}
We benchmark new atomic data against a selection of irradiances obtained from medium-resolution quiet-Sun spectra in the extreme ultraviolet (EUV), from 60 to $1040 \AA$. We used as a baseline the irradiances measured during solar minimum on 2008 April 14 by the prototype (PEVE) of the Solar Dynamics Observatory Extreme ultraviolet Variability Experiment (EVE). We took into account some inconsistencies in the PEVE data, using flight EVE data and irradiances we obtained from Solar and Heliospheric Observatory (SoHO) Coronal Diagnostics Spectrometer (CDS) data. We performed a differential emission measure and find overall excellent agreement (to within the accuracy of the observations, about 20\%) between predicted and measured irradiances in most cases, although we point out several problems with the currently available ion charge-state distributions. We used the photospheric chemical abundances of Asplund et al. (2009, ARA\&A, 47, 481). The new atomic data are nearly complete in this spectral range for medium-resolution irradiance spectra. Finally, we used observations of the active Sun in 1969 to show that the composition of the solar corona up to $1 \mathrm{MK}$ is nearly photospheric in this case as well. Variations of a factor of 2 are present for higher-temperature plasma, which is emitted within active regions. These results are in excellent agreement with our previous findings.
\end{abstract}

Key words. Sun: abundances - Sun: corona - Sun: UV radiation - line: identification - techniques: spectroscopic

\section{Introduction}

This paper is one of a series of studies where the extremeultraviolet (EUV) radiances and irradiances of the Sun are studied. One of the goals of these studies was the provision of well-calibrated EUV/UV irradiances of the quiet and active Sun to aid in the interpretation and modelling of stellar observations. As a first step on the modelling side, it is therefore important to assess the completeness and accuracy of current atomic data. This is the main aim of the present paper, where a large amount of recently calculated atomic rates are included. We focus on quiet-Sun irradiances, but also point out the differences with those of the more active Sun

The present benchmark also provides a test on ion chargestate distributions. When full-Sun irradiances are modelled, a continuous emission measure distribution is obtained, as is well known. This means that the predicted irradiances of lines of different ions that were formed at similar temperatures are expected to be close to the observed irradiances. Any discrepancies turn out to be mostly due to problems in the ion charge state calculations. Finally, this benchmark also provides an excellent way to measure the chemical abundance of the solar transition region and corona.

An earlier assessment was carried out within the SOLID ${ }^{1}$ project, which aimed at providing and estimating the solar spectral irradiance across all wavelengths. Within SOLID, a comparative study between the EUV/UV spectral radiances/irradiances as observed by several instruments and as obtained with

\footnotetext{
* The full Table A.1 is only available at the CDS via anonymous ftp to cdsarc.u-strasbg. fr (130.79.128.5) or via http://cdsarc . u-strasbg.fr/viz-bin/qcat?J/A+A/624/A36

1 http://projects.pmodwrc.ch/solid/
}

modelling using CHIANTI ${ }^{2}$ atomic data (Dere et al. 1997) was carried out. The earlier assessment was used to establish which atomic data needed improvement and which were missing, and it ultimately led to a significant update of the CHIANTI database, version 8 (Del Zanna et al. 2015a).

As most of the issues discussed in the previous series of papers is relevant to the analysis here, we provide a brief summary. Del Zanna et al. (2010; Paper I) presented a new radiometric calibration of the Solar and Heliospheric Observatory (SoHO) Coronal Diagnostics Spectrometer (CDS; Harrison et al. 1995) Normal Incidence Spectrometer (NIS), which included earlier calibrations (Del Zanna et al. 2001; Brekke et al. 2000). In Paper I, quiet-Sun NIS irradiances in 2008 were obtained and compared to those measured by a sounding rocket, flown on 2008 April 14 during the extended solar minimum, when the Sun was very quiet. The rocket carried a prototype (PEVE, see Woods et al. 2009) of the the Solar Dynamics Observatory (SDO) Extreme ultraviolet Variability Experiment (EVE, see Woods et al. 2012), and produced a medium-resolution EUV spectrum over a broad spectral range (60-1040 $)$ ). Very good agreement between the NIS and PEVE irradiances for most of the lines was found. However, problems in the PEVE irradiances in some of the strongest lines were found. This was surprising as the PEVE instrument was radiometrically calibrated on the ground (Chamberlin et al. 2007, 2009; Hock et al. 2010).

Our new SoHO CDS calibration allowed the first measurements of the EUV spectral irradiance along a solar cycle, from 1998 until 2010, which was presented in Del Zanna \& Andretta (2011; Paper II). The irradiances in lines formed below $1 \mathrm{MK}$ were shown to vary little, with the exception of the He lines.

\footnotetext{
2 wwW. chiantidatabase. org
} 
A new calibration for the most prominent line in the EUV, the He II $304 \AA$ resonance transition, was proposed. The change was significant and implied that most previously calibrated values were incorrect by about a factor of two. Our new NIS calibration was later confirmed by a sounding rocket flight, EUNIS (see Wang et al. 2011). Andretta \& Del Zanna (2014; Paper III) analysed in detail the radiances and limb-brightening in the main lines and their variation with solar cycle. Del Zanna et al. (2015b; Paper IV) compared the CDS irradiances with the count rates measured by the Solar EUV Monitor (SEM) first-order band (SEM 1). Del Zanna \& Andretta (2015; Paper V) extended and revised the CDS NIS calibration until 2014, when the instrument was switched off. The irradiances obtained from this analysis were then compared to those measured by PEVE, by SDO EVE, and by the Thermosphere Ionosphere Mesosphere Energetics Dynamics (TIMED) Solar EUV Experiment (SEE) EUV Grating Spectrograph (EGS) (Woods et al. 2005). Excellent agreement (to within a relative 10-20\%) with the EVE data (only during the 2010-2012 period) was found; however, the problems in the PEVE irradiances were confirmed, and several discrepancies with the TIMED EGS irradiances were also found.

The present assessment is the first of its kind in the EUV, as briefly pointed out below in Sect. 2 . Section 3 briefly describes the new atomic data used in the present paper, while Sect. 4 presents the quiet-Sun irradiances, a differential emission measure (DEM) modelling, and predicted spectra. Section 5 revisits older irradiance observations of the active Sun, and Sect. 6 draws the conclusions.

\section{Previous benchmark studies in the EUV}

Several assessments of atomic data in the EUV have been carried out previously, but on radiances in narrow wavelength ranges or in specific ions. For example, in a long series of papers starting with Del Zanna et al. (2004), the atomic data of several key ions have been benchmarked against laboratory and astrophysical spectra, providing many lacking identifications in the EUV, as reviewed in Del Zanna \& Mason (2018). Most of these identifications have been included in CHIANTI versions 7.1 and 8 .

A significant benchmark study based on the first SERTS rocket flight (Thomas \& Neupert 1994) was carried out by Young et al. (1998) in the $170-450 \AA$ range. The most complete previous assessment of atomic data for the EUV used SoHO CDS NIS and grazing incidence (GIS) radiances in the 150-780 $\AA$ range (Del Zanna 1999), but was limited by an uncertain CDS radiometric calibration and the use of older CHIANTI atomic data.

Landi et al. (2002a) also performed a benchmark of CHIANTI data for the coronal lines using off-limb observations from CDS NIS. An assessment of more recent CHIANTI atomic data in the 166-212 $\AA$ and 245-291 $\AA$ spectral ranges observed by the Extreme-ultraviolet Imaging Spectrometer (EIS, see Culhane et al. 2007) on board Hinode was carried out in Del Zanna (2012a) on coronal lines. Del Zanna (2009b) and Landi \& Young (2009) benchmarked CHIANTI atomic data for transition-region lines observed by EIS.

SoHO Solar Ultraviolet Measurements of Emitted Radiation (SUMER, Wilhelm et al. 1995, 700-1600 Å) quiet-Sun radiances were compared with earlier CHIANTI atomic data by Doschek et al. (1999), who found significant discrepancies. We note, however, that the lack of simultaneity in observing SUMER lines that are far apart in wavelength is a limiting issue for this type of studies, considering that the dominant lines are highly variable. An improvement was the benchmark of CHIANTI data for coronal lines using quiet-Sun off-limb SUMER observa- tions by Landi et al. (2002b), as these lines normally show little variability.

\section{Atomic data and ion and elemental abundances}

Within the UK APAP network ${ }^{3}$, we have carried out several large-scale structure and scattering $R$-matrix calculations over the past few years to produce atomic data for dozens of ions (see the review in Badnell et al. 2016). The present author has carried out a series of calculations, briefly listed in Del Zanna \& Mason (2018), that have greatly improved the atomic data for coronal ions. The main ions producing the majority of spectral lines in the EUV and soft X-rays are from iron: Fe viII (Del Zanna \& Badnell 2014), Fe Ix (Del Zanna et al. 2014), Fe x (Del Zanna et al. 2012a), Fe xi (Del Zanna \& Storey 2013), Fe xII (Del Zanna et al. 2012b), Fe XIII (Del Zanna \& Storey 2012), and Fe xIV (Del Zanna et al. 2015c). The UK APAP data have been included in CHIANTI version 8 (Del Zanna et al. 2015a).

For the assessment here, we use CHIANTI v. 8 as a baseline, together with a few minor changes that are being released within CHIANTI v.9 (Dere et al. 2019) and a large set of new atomic data that have been prepared for CHIANTI v.10 (Del Zanna et al., in prep.). These include APAP data for all the Be-like (Fernández-Menchero et al. 2014a) and Mg-like (Fernández-Menchero et al. 2014b) ions. New data for several ions are also included: S Iv (Del Zanna \& Badnell 2016a), Fe xIV (Del Zanna et al. 2015c), and Ni XII (Del Zanna \& Badnell (2016b). Radiative rates from a selection of sources were also added.

\subsection{Ion charge states at zero density}

During the course of this assessment, we have tested several ion charge state distributions (ion abundances), calculated assuming ionisation equilibrium at zero-density (the coronal approximation), that is, assuming that the entire population in an ion is in the ground state. The tables published in CHIANTI v.6 (Dere et al. 2009) are a significant improvement over previous tables because they include new ionisation rates (Dere 2007), radiative recombination rates (Badnell 2006), and dielectronic recombination rates (see Badnell et al. 2003 and the series of following papers).

The recombination rates for some ions were subsequently revised in CHIANTI v.7.1 (Landi et al. 2013). They mostly affected the charge state distribution of a few iron and nickel coronal ions, most notably Fe viII and Fe Ix. When we used the CHIANTI v.7.1 charge-state distributions, we obtained predicted irradiances that were about a factor of two higher than observed for both Fe vIII and Fe IX. It turned out that the large discrepancies were due to errors in the recombination rates of Fe vIII and Fe IX in CHIANTI. These were corrected and released in v.8.07, which we use here.

\subsection{Anomalous ions and ion charge states with density effects}

The Li- and Na-like ions give rise to some of the strongest lines in the EUV/UV spectral region. However, many of these ions are anomalous, in that the emission measures obtained from them are at odds with those obtained from ions of other isoelectronic sequences. This was first noted by Burton et al. (1971), although even earlier observations present the same problem, as described

\footnotetext{
www . apap-network . org
} 
in Del Zanna \& Mason (2018). Del Zanna et al. (2002) showed for the first time that the same problem is common in stars other than the Sun.

The issue of anomalous ions has largely been neglected in the literature, and there is still no obvious explanation, although several effects could be at play. Departures from ionisation equilibrium can significantly enhance some of the ions that have long ionisation/recombination times, as shown for instance in Bradshaw et al. (2004). Non-Maxwellian electron distributions tend to shift the formation temperature of the TR lines towards lower values, leading to an enhancement, as shown for Si iv by Dudík et al. (2014).

However, various effects related to the density of the plasma will always be present. One effect is a suppression of the dielectronic recombination, as described in Burgess \& Summers (1969). The authors developed a collisional-radiative modelling (CRM) that was further improved and implemented within the Atomic Data and Analysis Structure (ADAS), the consortium for fusion research. We have started a programme of new atomic calculations to model these and other (e.g. photo-ionisation) effects with up-to-date rates (Dufresne \& Del Zanna 2018), but for the present paper we have tested the ion fractions calculated using the effective rates as available in OPEN-ADAS ${ }^{4}$, which include density effects.

The helium lines (neutral and singly ionised) are also much stronger than predicted by large factors, an issue that has been discussed extensively in the literature. Various processes might lead to enhancements of these lines. Recombination following photo-ionisation from coronal radiation is one of them (see e.g. Andretta et al. 2003 and references therein). Non-equilibrium effects such as time-dependent ionisation (see e.g. Bradshaw et al. 2004) and diffusion processes (see e.g. Fontenla et al. 1993) are also important. A recent study by Golding et al. (2017) showed that time-dependent ionisation, recombination, and radiative transfer effects can indeed increase the intensities of the helium lines by a factor of 10 . We provide no attempt here to model lines from $\mathrm{He}$ or from $\mathrm{H}$, and point out that radiative transfer effects should in general be considered when some of the lines in neutral and singly ionised atoms are modelled.

\subsection{Coronal abundances of the quiet Sun are photospheric!}

Measurements of elemental abundances and temperatures are closely linked, since the DEM distribution can be used to describe the temperature distribution of the plasma and also to obtain relative elemental abundances (see the review of the methods in Del Zanna \& Mason 2018). It is well known that solar coronal abundances present variations and differ from the photospheric abundances, although there is a significant amount of controversy in the literature. The ratio of the coronal abundances of the low $(\leq 10 \mathrm{eV})$ first ionisation potential (FIP) elements vs. the high-FIP elements is often higher than the photospheric value (the so-called FIP bias). The significant revision of the abundances of several important high-FIP elements (e.g. carbon and oxygen) proposed by Asplund et al. (2009) is controversely discussed.

There is now sufficient evidence that chemical abundances in the quiet-Sun transition region are photospheric, while controversial results about the FIP bias in the corona have been published (see the reviews by Laming 2015; Del Zanna \& Mason 2018). Recent analyses of SoHO SUMER (Del Zanna \& DeLuca 2018) and UVCS (Del Zanna et al. 2018) using CHIANTI v.8

\footnotetext{
4 http://open.adas.ac.uk
}

of quiet-Sun areas show excellent agreement with the Asplund et al. (2009) photospheric abundances, mostly because the v.8 atomic data are significantly different compared to earlier ones. We therefore use these photospheric abundances for the present analysis. We return to the issue of chemical abundances below, when we present an analysis of the active Sun.

\section{Quiet-Sun irradiances}

We used the PEVE spectrum as the basis for our study of the quiet-Sun irradiances because it was taken during the extended solar minimum. The solar radio F10.7 flux was 69. The Sun was mainly featureless, with a polar coronal hole and a very small active region, which slightly affected a truly "quiet-Sun" measurement.

As shown in Paper V, the PEVE irradiances of several of the strong lines are much higher than those measured by CDS NIS, and they are also higher than the values measured in May 2010 by the in-flight EVE instrument (we considered version 5 data). We therefore studied the EVE daily irradiances during May-June 2010 to determine whether a relatively quiet period might be found. We considered the F10.7 radio flux and studied the variation of the EVE irradiances. We selected 2010 May 16 as one of the best dates. Unfortunately, the Sun already had five small active regions on the visible side, and indeed all the irradiances of the lines formed above $1 \mathrm{MK}$ are significantly enhanced in the 2010 May 16 EVE spectrum compared to the PEVE spectrum, even though the F10.7 radio flux was only 70 . This comparison incidentally clearly shows that the F10.7 radio flux is not a good indicator of very low levels of solar activity. We measured the irradiances of both the EVE and PEVE lines subtracting a background, but we note that this background subtraction only affects the measurements of the main lines by at most $10 \%$, as shown in Paper V.

The discrepancies between the PEVE and EVE observations are sometimes significant (50\%), well above the quoted uncertainties, and cannot be due to solar variability for lines formed below $1 \mathrm{MK}$. All the low-temperature emission lines (except the helium lines) vary very slightly during the solar cycle, as shown in Paper II and Paper III. The differences can only be due to calibration problems. To aid in the assessment, we considered all the historical records of EUV irradiances, as carried out previously in Paper II. We also considered our CDS NIS irradiances during solar minimum to assess when the PEVE values were reasonable. In a few cases, we replaced the PEVE irradiances with the EVE measurements. The irradiances of a selection of EUV lines are given in Table 1, where other measurements for some of the cooler lines are also provided. We only list the measurements that we regard as relatively accurate: our CDS NIS measurements of 2008 September 22, and the irradiances published by Malinovsky \& Heroux (1973) and Heroux et al. (1974). The F10.7 radio flux on 2008 September 22 was 69.6, and the Sun only had a small active region, or in other words, it was very quiet. Malinovsky \& Heroux (1973) published a calibrated spectrum in the $50-300 \AA$ range with a medium resolution $(0.25 \AA)$, taken with a grazing-incidence spectrometer flown on a rocket on 1969 April 4, when the F10.7 flux was 177.3 , that is, when the Sun was active, and active regions and flares contributed significantly. Heroux et al. (1974) provided irradiances that were also obtained from a sounding rocket flown on 1972 August 23 when the F10.7 flux was 120, that is, when the Sun was moderately active. A more extended list of EUV quiet-Sun irradiances is provided in Table A.1 in the Appendix. 
Table 1. Observed and predicted quiet-Sun EUV irradiances for transition region and coronal lines for increased formation temperature.

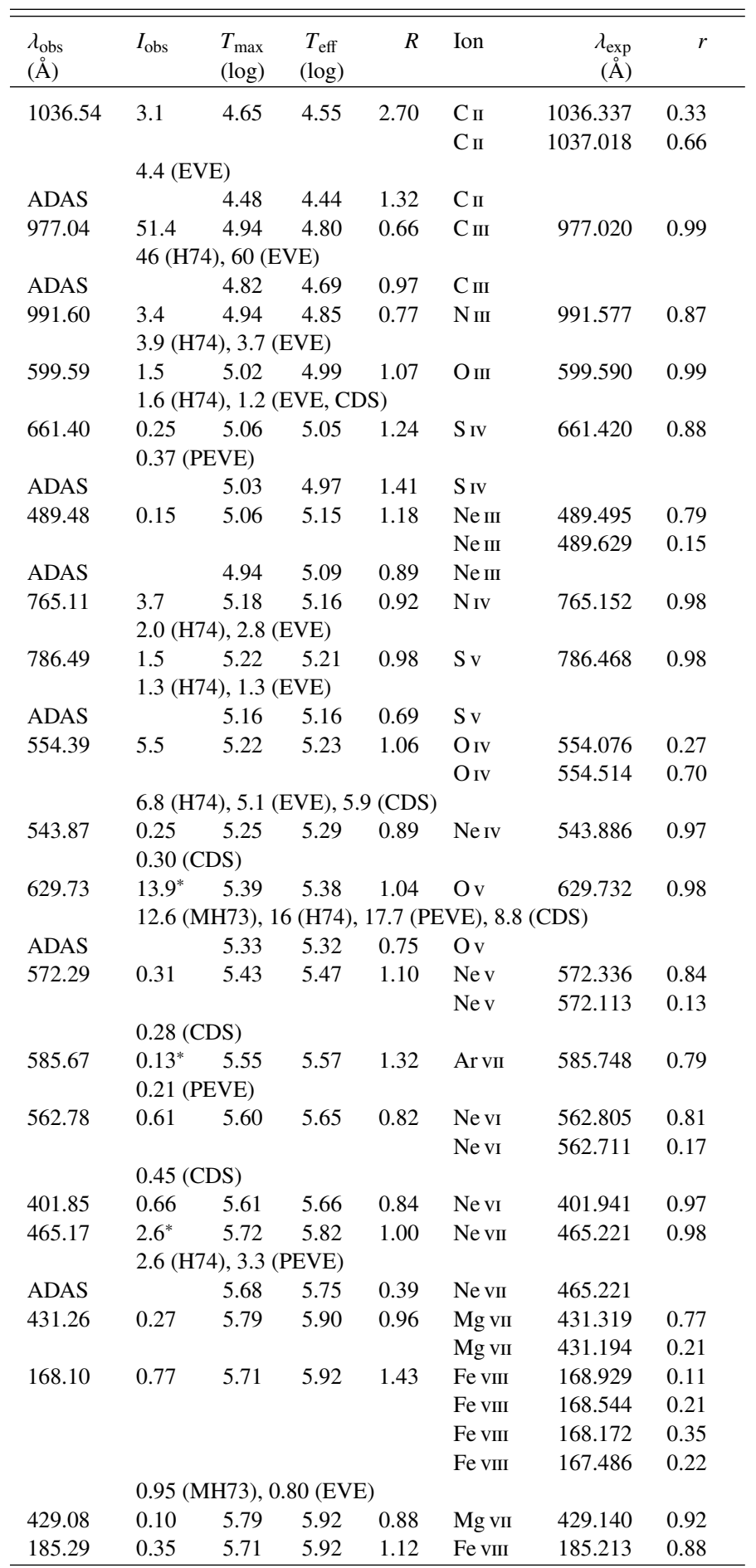

Notes. Each line lists the observed wavelengths $\lambda_{\text {obs }}(\AA)$, the measured irradiances $I_{\mathrm{obs}}\left(10^{8}\right.$ photons $\left.\mathrm{cm}^{-2} \mathrm{~s}^{-1} \operatorname{arcsec}^{-2}\right)$, the maximum and effective temperature (log values, in K; see text) $T_{\max }$ and $T_{\text {eff }}$, the ratio $R$ between the predicted and observed values, the main contributing ion and CHIANTI wavelength $\lambda_{\exp }(\AA)$, and the fractional contribution $r$ (only contributions greater than $10 \%$ are shown) to the blend. $I_{\text {obs }}$ are prototype EVE (PEVE) irradiances, while those with an asterisk $\left({ }^{*}\right)$ are the EVE values of 2010 May 16. Measured irradiance from MH73: Malinovsky \& Heroux (1973); H74: Heroux et al. (1974) and CDS (2008 Sept 22) are also listed in some cases. The lines with ADAS indicate the values obtained using the OPEN-ADAS ion charge-state distributions.
Table 1. continued.

\begin{tabular}{|c|c|c|c|c|c|c|c|}
\hline $\begin{array}{l}\lambda_{\mathrm{obs}} \\
(\AA)\end{array}$ & $I_{\mathrm{obs}}$ & $\begin{array}{l}T_{\max } \\
(\log )\end{array}$ & $\begin{array}{r}T_{\text {eff }} \\
(\log )\end{array}$ & $R$ & Ion & $\begin{array}{r}\lambda_{\exp } \\
(\AA) \\
\end{array}$ & $r$ \\
\hline & 0.44 & MH73), & $.26(\mathrm{E})$ & & & & \\
\hline \multirow[t]{3}{*}{278.40} & 0.33 & 5.80 & 5.92 & 1.05 & Mg VII & 278.404 & 0.63 \\
\hline & & & & & Si VII & 278.450 & 0.30 \\
\hline & 0.34 & MH73), & $.36\left(\mathrm{H}^{7}\right.$ & 4), 0.27 & (EVE) & & \\
\hline \multirow[t]{3}{*}{681.81} & 0.15 & 5.94 & 5.93 & 1.11 & Na Ix & 681.719 & 0.78 \\
\hline & & & & & $S_{\text {III }}$ & 681.488 & 0.18 \\
\hline & 0.25 & EVE) & & & & & \\
\hline \multirow[t]{3}{*}{275.42} & 0.35 & 5.80 & 5.93 & 1.08 & Si VII & 275.361 & 0.84 \\
\hline & & & & & Si VII & 275.676 & 0.14 \\
\hline & 0.28 & MH73), & $.25\left(\mathrm{H}^{7}\right.$ & 4), 0.26 & (EVE) & & \\
\hline ADAS & & 5.73 & 5.88 & 0.48 & Si VII & & \\
\hline 466.10 & 0.45 & 5.81 & 5.93 & 0.68 & Ca Ix & 466.240 & 0.98 \\
\hline 411.15 & 0.23 & 5.87 & 5.94 & 0.53 & Na VIII & 411.171 & 0.93 \\
\hline \multirow[t]{2}{*}{780.31} & 2.1 & 5.80 & 5.96 & 1.01 & Ne VIII & 780.385 & 0.95 \\
\hline & 1.8 & H73) 1. & (H74), & $2.5(\mathrm{PE}$ & VE) & & \\
\hline ADAS & & 5.74 & 5.92 & 0.79 & Ne vIII & & \\
\hline \multirow{2}{*}{315.07} & 1.1 & 5.91 & 5.98 & 1.03 & $\mathrm{Mg}_{\text {VIII }}$ & 315.015 & 0.97 \\
\hline & 1.35 & CDS) & & & & & \\
\hline 430.41 & 0.75 & 5.91 & 5.98 & 0.84 & $\mathrm{Mg}_{\text {VIII }}$ & 430.454 & 0.97 \\
\hline \multirow{2}{*}{170.98} & 7.0 & 5.91 & 6.00 & 0.95 & Fe IX & 171.073 & 0.99 \\
\hline & 4.4 (1 & H73), 4 & $2(\mathrm{H} 74)$ & $3.95(\mathrm{H}$ & VE) & & \\
\hline \multirow[t]{2}{*}{319.86} & 1.4 & 5.94 & 6.01 & 0.89 & Si vIII & 319.840 & 0.98 \\
\hline & 1.38 & CDS) & & & & & \\
\hline ADAS & & 5.87 & 5.97 & 0.66 & Si VIII & & \\
\hline \multirow[t]{2}{*}{706.05} & 0.52 & 5.99 & 6.02 & 0.78 & $\mathrm{Mg}_{\mathrm{IX}}$ & 706.060 & 0.95 \\
\hline & 0.34 & EVE) & & & & & \\
\hline \multirow[t]{2}{*}{557.60} & 0.26 & 5.89 & 6.03 & 1.30 & $\mathrm{Cax}$ & 557.766 & 0.99 \\
\hline & 0.21 & CDS) & & & & & \\
\hline \multirow[t]{2}{*}{368.10} & $5.8^{*}$ & 5.99 & 6.03 & 0.86 & $\operatorname{Mg}_{\text {IX }}$ & 368.071 & 0.96 \\
\hline & $6.2(\mathrm{l}$ & 74) 8.1 & PEVE), & $5.8(\mathrm{CD}$ & & & \\
\hline 272.05 & 0.42 & 5.28 & 6.03 & 0.79 & Six & 271.992 & 0.75 \\
\hline \multirow{2}{*}{443.80} & 0.25 & 5.99 & 6.03 & 1.00 & $\mathrm{Mg}_{\mathrm{IX}}$ & 443.404 & 0.16 \\
\hline & & & & & $\mathrm{Mg}_{\text {IX }}$ & 443.973 & 0.78 \\
\hline \multirow[t]{3}{*}{198.51} & 0.26 & 5.96 & 6.04 & 0.84 & S VIII & 198.554 & 0.65 \\
\hline & & & & & $\mathrm{Fe} \mathrm{xI}$ & 198.538 & 0.27 \\
\hline & 0.23 & MH73), & $.24(\mathrm{E})$ & & & & \\
\hline \multirow[t]{3}{*}{345.13} & 0.90 & 6.06 & 6.06 & 0.72 & Si IX & 345.121 & 0.74 \\
\hline & & & & & Si IX & 344.954 & 0.21 \\
\hline & 1.37 & CDS) & & & & & \\
\hline \multirow[t]{2}{*}{174.57} & 3.7 & 6.05 & 6.06 & 1.03 & $\mathrm{Fex}$ & 174.531 & 0.98 \\
\hline & $4.6(\mathrm{I}$ & H73), 4 & $1(\mathrm{H} 74)$ & $3.8(\mathrm{E}$ & & & \\
\hline 624.94 & 2.1 & 6.07 & 6.07 & 0.76 & $\operatorname{Mg} x$ & 624.968 & 0.92 \\
\hline & 1.4 & DS) & & & & & \\
\hline 148.38 & 0.38 & 6.11 & 6.08 & 1.14 & $\mathrm{Ni}$ XI & 148.377 & 0.99 \\
\hline & 0.59 & H74), 0. & 5 (EVE & & & & \\
\hline 188.29 & 2.3 & 6.13 & 6.09 & 1.08 & $\mathrm{Fe} \mathrm{XI}$ & 188.216 & 0.50 \\
\hline & & & & & $\mathrm{Fe} \mathrm{XI}$ & 188.299 & 0.31 \\
\hline & & & & & Fe IX & 188.493 & 0.13 \\
\hline 178.09 & 0.14 & 6.12 & 6.10 & 0.78 & Fe XI & 178.058 & 0.91 \\
\hline 180.41 & 2.7 & 6.13 & 6.10 & 1.07 & $\mathrm{Fe} \mathrm{XI}$ & 180.401 & 0.84 \\
\hline 264.30 & 0.38 & 6.18 & 6.13 & 0.71 & $\mathrm{Sx}$ & 264.231 & 0.98 \\
\hline 152.05 & 0.11 & 6.24 & 6.14 & 0.80 & Ni XII & 152.151 & 0.94 \\
\hline & 0.36 & H74), 0. & 3 (EVE & & & & \\
\hline 195.09 & 1.4 & 6.20 & 6.15 & 0.96 & Fe XII & 195.119 & 0.95 \\
\hline 202.10 & 0.96 & 6.25 & 6.15 & 0.92 & Fe XIIII & 202.044 & 0.64 \\
\hline & & & & & $\mathrm{Fe} \mathrm{XI}$ & 202.424 & 0.12 \\
\hline 211.40 & 0.33 & 6.29 & 6.19 & 0.97 & Fe XIV & 211.317 & 0.75 \\
\hline & & & & & Ni XI & 211.430 & 0.12 \\
\hline 499.41 & 0.34 & 6.29 & 6.20 & 0.95 & Si XII & 499.406 & 0.94 \\
\hline ADAS & & 6.24 & 6.18 & 2.14 & Si XII & & \\
\hline 284.12 & 0.46 & 6.34 & 6.24 & 1.05 & Al Ix & 284.042 & 0.20 \\
\hline & & & & & $\mathrm{Fe} x \mathrm{v}$ & 284.163 & 0.76 \\
\hline
\end{tabular}

\subsection{DEM and predicted irradiances}

A DEM modelling was carried out to explore a range of parameters. We used the Del Zanna (1999) method, in which the DEM is modelled as a spline distribution. The intensity of the vast majority of the strongest spectral lines is largely independent of the density, but some transition region lines are sensitive to 
the varying density in the lower part of the solar atmosphere. An example is the O v multiplet at $760 \AA$. We adopted a model with a constant pressure of $5 \times 10^{14} \mathrm{~cm}^{-3} \mathrm{~K}^{-1}$ and the CHIANTI v.8.07 zero-density ion abundances. The resulting volume DEM is shown in Fig. 1. To indicate how well the DEM is constrained, we also plot the ratio of the predicted vs. observed irradiance in Fig. 1, multiplied by the DEM value at the effective temperature,

$$
T_{\text {eff }}=\frac{\int T G(T) \operatorname{DEM}(T) \mathrm{d} T}{\int G(T) \operatorname{DEM}(T) \mathrm{d} T},
$$

which is an average temperature that is more indicative of where a line is formed. This is often quite different than $T_{\max }$, the temperature where the $G(T)$ of a line has a maximum.

For comparison, we show in Fig. 1 the DEM obtained from the CDS NIS irradiances of 2008 Sept. 22 (cf. Paper IV). There is overall agreement, as expected. We stress that as noted in Paper IV, all the DEM distributions that we have obtained from NIS along the solar cycle are all similar below $1 \mathrm{MK}$ because of the little variation in the irradiances of the cooler lines.

The irradiances predicted using the DEM and the zerodensity ion abundances are also listed in Table 1. In most of the cases, we obtain similar irradiances using the same DEM and the ion abundances calculated from OPEN-ADAS at a constant pressure of $5 \times 10^{14} \mathrm{~cm}^{-3} \mathrm{~K}^{-1}$, but we note that the density effects tend to shift the formation temperature of a transitionregion ion towards lower values. However, significant discrepancies are present in several cases. We only list the main discrepancies in Table 1.

Our results in terms of anomalous ions differ in some respect from earlier results. The irradiances of the higher-temperature lines, Li-like Ne VIII, Na Ix, Mg X, and Si XII, are well represented, unlike in many previous reports. The reasons might be the better instrument calibration and that we analysed the solar irradiance during solar minimum. When active regions are present, nonequilibrium effects are likely to become stronger.

For some low-temperature ions such as $\mathrm{C}$ II and $\mathrm{C}$ III (as well as the anomalous ion $\mathrm{C}$ IV, not shown here), the OPEN-ADAS rates provide a significant improvement over the zero-density rates. However, significant discrepancies are present in many cases. The most obvious differences are those for the strong $\mathrm{Ne}$ VII, Si vII, and Si XII lines, where the predicted irradiances are different by a factor of two. The problems also persist when the ADAS data are used to infer a DEM. It is impossible to ascertain the reasons for these discrepancies because no information on the basic rates used in the ADAS CRM is available.

\subsection{Quiet-Sun EUV spectra}

The DEM and the same set of parameters as used for the inversion were then used to produce a modelled spectrum that was to be compared to the observed one, to assess the completeness in the atomic data. The PEVE quiet-Sun spectrum of 2008 is shown in black in Fig. 2, and the quiet-Sun EVE spectrum on 2010 May 16 is shown in grey. Figure 2 shows that any small solar activity significantly affects the solar spectrum at most wavelengths. The predicted line profiles are approximated with Gaussians and are shown in red in Fig. 2, and the positions and intensities of the main contributing lines are shown by vertical blue lines. We recall that several PEVE irradiances are not correct, and that lines from $\mathrm{H}$ and $\mathrm{He}$ are notoriously difficult to model. We now briefly summarise the main findings for each wavelength region.

\subsubsection{Soft X-rays: $60-160 \AA$}

As shown in Fig. 2, relatively good agreement with the PEVE data is found, although further work is still needed in this relatively unexplored spectral region. When we calculated the atomic data for the coronal iron ions, we were surprised to find that many lines have increased intensities when compared to distorted wave calculations (available since CHIANTI v.7.1, see Landi et al. 2013). This was due to resonance enhancements in the excitation rates and cascading effects. These new atomic data and identifications have been included in CHIANTI v. 8 and allowed the first identifications of the strongest iron coronal lines in this spectral region, using solar and laboratory spectra with much higher resolution (Del Zanna 2012b).

\subsection{2. $160-310 \AA$ spectral region}

Most of the 160-290 $\AA$ spectral region is now well understood, after several studies based on the Hinode EIS spectra (cf. Del Zanna 2012a), and the new atomic data mentioned before. The agreement between theory and observations is within a few percent, with the exception of one of the Fe vIII lines at $168.7 \AA$ and the strong Fe Ix resonance line at $171 \AA$. As we described previously, the disagreement is much larger when CHIANTI ion abundances earlier than 8.0.7 are considered.

We note that the PEVE irradiances of the Fe VIII lines around $168 \AA$ are in good agreement with the EVE and those of Malinovsky \& Heroux (1973), and several weaker Fe vIII lines at longer wavelengths are very well reproduced. All the Fe vIII EUV lines were benchmarked against high-resolution solar and laboratory observations (Del Zanna 2009a); the agreement was good.

We also note an inconsistency in the measured irradiances of the Fe Ix $171 \AA$ line. As shown in Table 1, the PEVE irradiance is $7.0\left(10^{8}\right.$ photons $\left.\mathrm{cm}^{-2} \mathrm{~s}^{-1} \operatorname{arcsec}^{-2}\right)$, but the EVE v.5 value is much lower, 3.95. The Malinovsky \& Heroux (1973) value is 4.4. Excellent agreement with theory is found when the PEVE value is used. We note that all the other Fe Ix lines at longer wavelengths, most notably the two density-sensitive lines at 241.74 and $244.91 \AA$, are also very well reproduced.

In an earlier benchmark (Del Zanna et al. 2011), we showed that several coronal lines in the 205-240 A are still unidentified, which means that further work is needed in this spectral region. This is also shown in Table A.1, although these missing lines are not very strong in solar irradiance spectra. Except for this region, good agreement is found, as Fig. 2 shows. One exception is the series of He II lines at 304, 256, and $243 \AA$.

\subsection{3. $310-380 \AA ̊$ spectral region}

The 310-380 $\AA$ spectral region is also well reproduced because significant effort to improve the atomic data was produced before the launch of SoHO, as shown by the earlier atomic benchmark studies using the SoHO CDS NIS 1 spectra (Del Zanna 1999). Many of the lines in this spectral region are due to coronal iron ions, for which the new atomic data in CHIANTI v. 8 are a significant improvement. Several iron lines in this band show enhancements of about $30 \%$. This is caused by resonance enhancement and cascading effects that increase the decays from the lower levels that produce the lines in this spectral region.

\subsubsection{0-510 A spectral region}

The quiet-Sun spectrum in the $380-510 \AA$ region is dominated by transition region lines from $\mathrm{Ne}$ and $\mathrm{Mg}$ ions. This spectral 


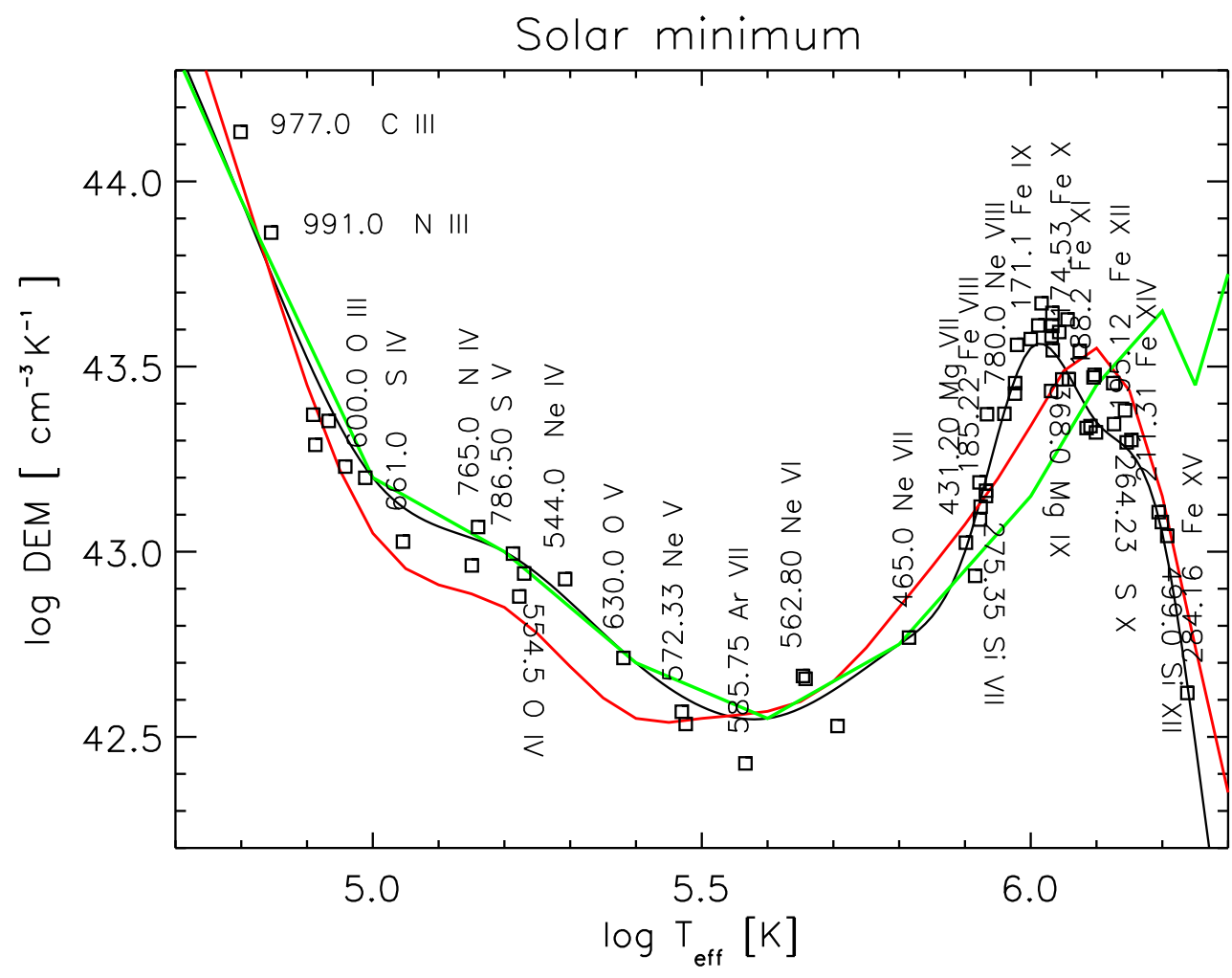

Fig. 1. Volume DEM distribution of the quiet Sun. The points indicate the ratio of the predicted vs. observed irradiance, multiplied by the DEM value at the effective temperature. The labels indicate the wavelength $(\AA)$ and the main ion. The red line indicates the DEM obtained from the CDS NIS measurements of 2008 Sept 22, while the green line the DEM obtained from the active Sun using the Malinovsky \& Heroux (1973) irradiances. region is also well understood because various studies based on the SoHO CDS GIS spectra were performed (Del Zanna 1999) and because the atomic data for the ions in this spectral range are somewhat easier to calculate. Figure 2 shows a very good agreement between observed and predicted irradiances. We note that the neutral helium continuum (with the edge at $504 \AA$ ) cannot be modelled properly without taking radiative transfer effects into account.

\subsection{5. $510-630 \AA$ spectral region}

The 510-630 A spectral region is also well known and complete in terms of atomic data because SoHO CDS NIS 2 spectra have been used to benchmark the atomic data (Del Zanna 1999), and most of the ions are low charge. Very good agreement is found, with the exception of the series of He I lines.

\subsection{6. $630-780 \AA$ spectral region}

The 630-780 $\AA$ spectral region has been studied using CDS GIS spectra (Del Zanna 1999). Good overall agreement between observed and predicted line irradiances is found. We note that the intensities of the $\mathrm{O}_{\text {II }} 718 \AA$ line and of the $\mathrm{O} v$ multiplet at $760 \AA$ are very sensitive to the temperatures and densities of the model. With the current atomic data, the $\mathrm{O} v$ multiplet is well represented, but the $\mathrm{O}_{\text {II }}$ line is not. Using the ADAS ion abundances for $\mathrm{O}$ II does not improve the comparison. We also note that the hydrogen continuum (with the edge at $912 \AA$ ) cannot be modelled properly without taking radiative transfer effects into account.

\subsection{7. $780-912 \AA$ spectral region}

The few $\mathrm{O}$ III and $\mathrm{O}$ IV in this wavelength region are well represented (cf. Table A.1), but all the $\mathrm{O}_{\text {II }}$ lines are incorrectly modelled (also using the ADAS ion abundances).

\subsubsection{2-1045 $\AA$ spectral region}

The $\mathrm{C}_{\text {III }}$ resonance line at $977 \AA$ is relatively well represented with the CHIANTI v.8.0.7 ion fractions, although the ADAS ion abundances improve agreement. The $\mathrm{N}_{\text {III }} 991.57 \AA$ line is also well represented (cf. Table A.1). The doublet at 1031.9, 1037.6 from the anomalous ion $\mathrm{OVI}_{\mathrm{VI}}$ is under-predicted by more than a factor of 2. Only a small improvement is obtained using the ADAS ion abundances.

\section{Active-Sun irradiances and chemical abundances}

The Laming et al. (1995) study, based on the Malinovsky \& Heroux (1973) spectrum, is often cited in the literature to support the argument that the solar corona has an FIP bias of about 4. However, Laming et al. (1995) clearly stated that nearly photospheric abundances were found when considering lines formed in the transition region, up to $1 \mathrm{MK}$. The FIP bias of 3-4 was obtained from higher-temperature lines. Clearly, this hot emission would be significantly biased by the active regions and not by the quiet Sun, where almost no emission above $1 \mathrm{MK}$ is present.

To interpret the full-Sun spectra such as those of Malinovsky \& Heroux (1973), we therefore need a good understanding of the abundances in active regions. Controversial results about the FIP bias in the hot ( $3 \mathrm{MK}$ ) cores of active regions have been published in the literature. However, a recent revision of EUV (Hinode/EIS) and X-ray (SMM/FCS) measurements of several active regions has indicated a remarkable consistency, with an FIP bias of about 3.2 (Del Zanna 2013b; Del Zanna \& Mason 2014). Del Zanna (2013b) also showed that it must be the low-FIP elements that are enhanced by at least a factor of 3, compared to the photospheric values. We note that the Del Zanna (2013b) results were obtained using a new Hinode EIS calibration (Del Zanna 2013a).

Considering the significant updates in atomic data since the Laming et al. (1995) study and the changes in the photospheric 

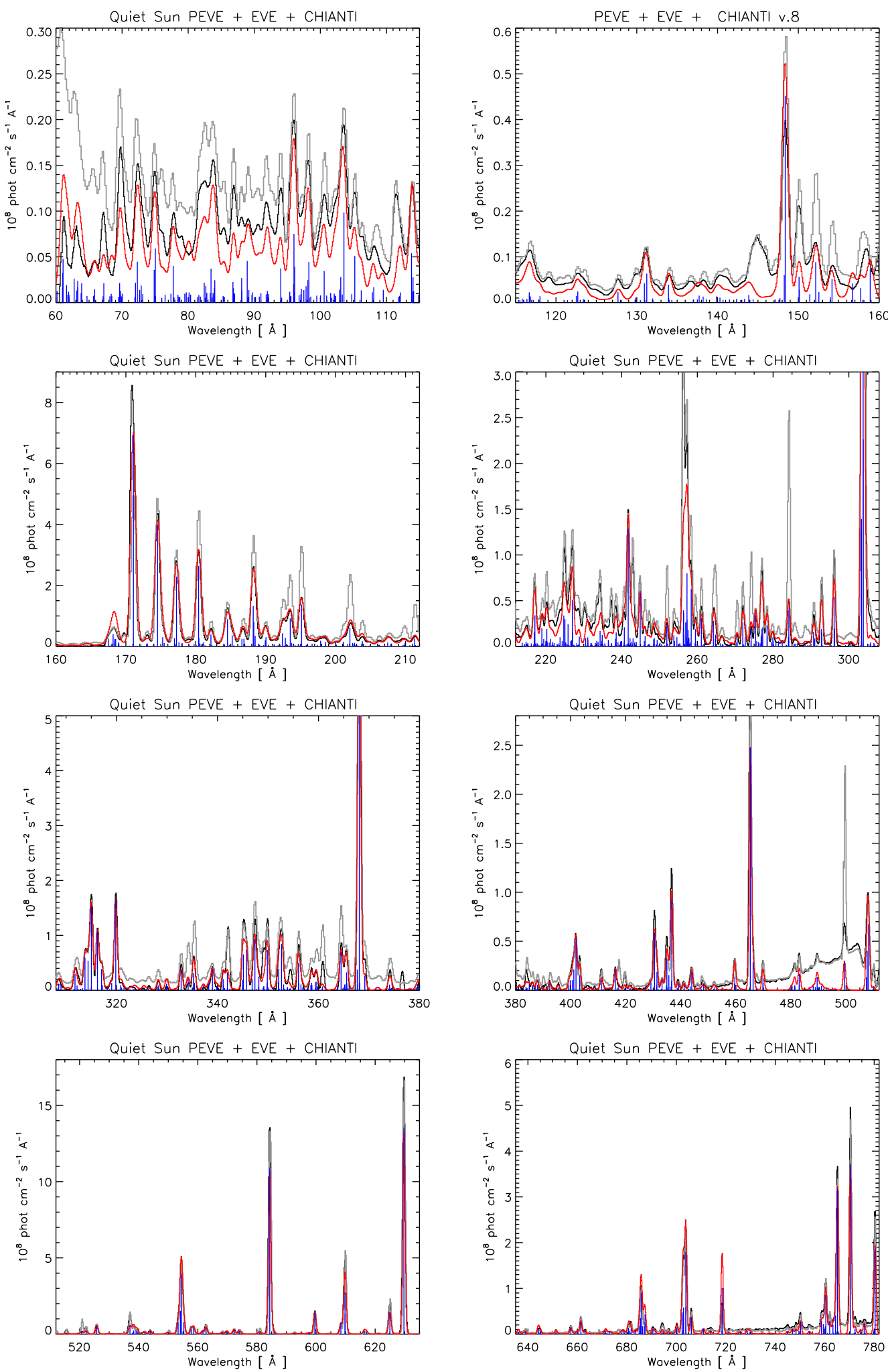

Fig. 2. Observed PEVE quiet-Sun spectrum in 2008 (black), with the observed EVE spectrum on 2010 May 16 (grey) and the predicted spectrum (red). The positions and intensities of the main contributing lines are shown by vertical blue lines.

abundances recommended by Asplund et al. (2009), it is worth reconsidering the well-calibrated Malinovsky \& Heroux (1973) EUV irradiances. We have chosen a selection of lines in the Malinovsky \& Heroux (1973) EUV spectrum, avoiding the lines that are strongly density dependent. We note that in several cases, the authors had deblended the intensities of lines close in wavelength. The spectrum must have been obtained after or during flaring emission because the Fe xvIII line at $93.9 \AA$ was relatively strong. We have taken the deblended value as recommended by Malinovsky \& Heroux (1973), but applied a correction factor of 2 to its irradiance, following the problems in the soft X-rays described in Del Zanna (2012b). With this 


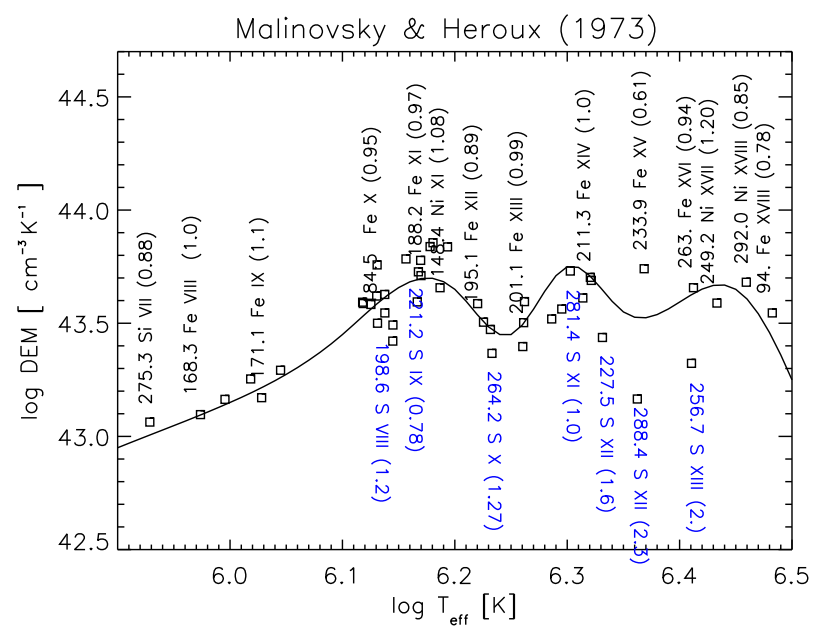

Fig. 3. DEM distribution obtained from active-Sun Malinovsky \& Heroux (1973) irradiances. The points indicate the ratio of the predicted vs. observed irradiance, multiplied by the DEM value at the effective temperature. The labels indicate the wavelength $(\AA)$, the main ion, and the ratio of the predicted vs. observed irradiance (in brackets).

correction, good agreement between predicted and observed irradiance is obtained.

We used the set of atomic data described above, the CHIANTI v.8.07 zero-density ion abundances, and the Asplund et al. (2009) photospheric abundances. We assumed a constant density of $1 \times 10^{9} \mathrm{~cm}^{-3}$ for the calculation of the line emissivities.

The DEM, shown in Fig. 3, is significantly different than the quiet-Sun DEM for temperatures higher than $1 \mathrm{MK}$, as expected. The predicted irradiances are shown in Table 2. The analysis confirms our results described above, in particular, that up to $1.5 \mathrm{MK}$, the chemical abundances are nearly photospheric (within $30 \%$, cf. S viII, S Ix, S x, and S xi vs. the low-FIP iron ions). The $S x$ abundance is well constrained because the $264.2 \AA$ line is relatively strong and strong iron lines are formed at the same temperature.

We clearly see, however, the effect of the presence of active regions on these active-Sun irradiances. The iron/nickel lines require two secondary peaks at higher temperatures. The abundance of sulphur deviates by a factor of about 2 around $2 \mathrm{MK}$, as seen in S XII and S XIII. The S XII abundance is well constrained relative to the Fe XIV resonance line at $211.3 \AA$, which is formed at similar temperatures. The $\mathrm{S}$ XIII abundance is well constrained relative to the Fexvi emission. These results are in excellent agreement with our active region Hinode EIS results (Del Zanna 2012a), but not with those of Brooks et al. (2015) nor with the previous analysis of Laming et al. (1995).

Finally, we note that the DEM shown in Fig. 3 is misleading because we obtained it assuming constant abundances of the low-FIP iron/nickel elements. Consequently, the sulphur abundance at $2 \mathrm{MK}$ appears twice lower than photospheric.

\section{Summary and conclusions}

Overall, we found a remarkable agreement to within a relative $20 \%$ accuracy between predicted and observed quiet-Sun line irradiances. We have considered the spectra that were measured during the extended solar minimum in 2008 by the EVE prototype, but highlighted several cases where the prototype values appear to suffer from calibration problems, by comparing them with in-flight EVE data and our well-calibrated SoHO CDS irradiances (Del Zanna \& Andretta 2015). Comparisons of the
Table 2. Observed and predicted EUV irradiances from the Malinovsky \& Heroux (1973) spectrum (same as Table 1).

\begin{tabular}{|c|c|c|c|c|c|c|c|}
\hline$\lambda_{\mathrm{obs}}$ & $I_{\mathrm{obs}}$ & $T_{\max }$ & $T_{\mathrm{eff}}$ & $R$ & Ion & $\lambda_{\exp }$ & $r$ \\
\hline 629.73 & 12.7 & 5.39 & 5.38 & 1.14 & $\mathrm{Ov}$ & 629.732 & 0.98 \\
\hline 275.42 & 0.28 & 5.79 & 5.93 & 0.88 & Si viI & 275.361 & 0.98 \\
\hline \multirow[t]{3}{*}{168.10} & 0.96 & 5.71 & 5.97 & 1.00 & Fe vIII & 168.544 & 0.19 \\
\hline & & & & & Fe vIII & 168.172 & 0.31 \\
\hline & & & & & Fe vIII & 167.486 & 0.19 \\
\hline \multirow[t]{2}{*}{186.60} & 0.25 & 5.71 & 6.02 & 0.87 & Ca XIv & 186.610 & 0.11 \\
\hline & & & & & Fe viII & 186.598 & 0.86 \\
\hline 170.98 & 4.4 & 5.90 & 6.03 & 1.11 & Fe IX & 171.073 & 0.99 \\
\hline \multirow[t]{3}{*}{278.40} & 0.34 & 5.79 & 6.04 & 0.93 & P xII & 278.286 & 0.17 \\
\hline & & & & & Mg VII & 278.404 & 0.52 \\
\hline & & & & & $\mathrm{Si}$ VII & 278.450 & 0.25 \\
\hline 174.57 & 4.6 & 6.05 & 6.12 & 0.87 & $\mathrm{Fe} x$ & 174.531 & 0.98 \\
\hline 177.20 & 2.6 & 6.05 & 6.12 & 0.88 & $\mathrm{Fex}$ & 177.240 & 0.98 \\
\hline 184.46 & 1.0 & 6.05 & 6.13 & 0.95 & Fex & 184.537 & 0.93 \\
\hline \multirow[t]{2}{*}{296.15} & 0.94 & 6.05 & 6.13 & 0.92 & Si IX & 296.211 & 0.23 \\
\hline & & & & & Si IX & 296.113 & 0.74 \\
\hline 225.00 & 0.51 & 6.06 & 6.13 & 0.67 & Si IX & 225.025 & 0.98 \\
\hline \multirow[t]{2}{*}{198.51} & 0.23 & 5.96 & 6.13 & 1.22 & S VIII & 198.554 & 0.43 \\
\hline & & & & & Fe XI & 198.538 & 0.48 \\
\hline \multirow[t]{2}{*}{185.29} & 0.44 & 5.72 & 6.14 & 0.97 & $\mathrm{Ni}$ xVI & 185.230 & 0.29 \\
\hline & & & & & Fe VIII & 185.213 & 0.63 \\
\hline \multirow[t]{2}{*}{609.90} & 6.0 & 5.21 & 6.14 & 1.17 & $\operatorname{Mg} x$ & 609.794 & 0.79 \\
\hline & & & & & OIV & 609.830 & 0.20 \\
\hline 770.41 & 3.0 & 5.80 & 6.14 & 1.66 & $\mathrm{Ne}$ VIII & 770.428 & 1.00 \\
\hline 780.31 & 1.8 & 5.80 & 6.14 & 1.41 & Ne VIII & 780.385 & 1.00 \\
\hline 221.24 & 0.14 & 6.06 & 6.16 & 0.78 & $S_{\text {IX }}$ & 221.241 & 0.98 \\
\hline 178.09 & 0.18 & 6.13 & 6.17 & 1.26 & Fe XI & 178.058 & 0.95 \\
\hline 180.41 & 5.3 & 6.13 & 6.17 & 0.93 & Fe XI & 180.401 & 0.87 \\
\hline 272.05 & 0.75 & 5.29 & 6.17 & 0.83 & Six & 271.992 & 0.84 \\
\hline \multirow[t]{2}{*}{188.29} & 3.8 & 6.13 & 6.17 & 0.97 & $\mathrm{Fe} \mathrm{XI}$ & 188.216 & 0.60 \\
\hline & & & & & Fe xI & 188.299 & 0.36 \\
\hline \multirow[t]{2}{*}{290.70} & 0.31 & 6.05 & 6.18 & 0.73 & Si IX & 290.687 & 0.75 \\
\hline & & & & & Fe XIV & 290.749 & 0.15 \\
\hline \multirow[t]{2}{*}{223.70} & 0.27 & 6.07 & 6.18 & 0.70 & Si IX & 223.744 & 0.60 \\
\hline & & & & & Fe XIII & 223.778 & 0.21 \\
\hline 148.38 & 0.72 & 6.11 & 6.19 & 1.08 & $\mathrm{Ni}$ XI & 148.377 & 0.99 \\
\hline 261.06 & 0.89 & 6.15 & 6.19 & 0.69 & Si $x$ & 261.056 & 0.97 \\
\hline 195.09 & 4.8 & 6.19 & 6.22 & 0.89 & Fe XII & 195.119 & 0.92 \\
\hline 624.94 & 2.8 & 6.06 & 6.23 & 1.00 & $\operatorname{Mg} x$ & 624.968 & 0.99 \\
\hline \multirow[t]{2}{*}{259.50} & 0.68 & 6.18 & 6.23 & 1.01 & $\mathrm{Sx}$ & 259.497 & 0.80 \\
\hline & & & & & Fe XII & 259.418 & 0.15 \\
\hline 264.20 & 0.65 & 6.18 & 6.23 & 1.27 & Sx & 264.231 & 0.98 \\
\hline \multirow[t]{2}{*}{239.80} & 0.17 & 6.19 & 6.26 & 1.25 & S XI & 239.817 & 0.63 \\
\hline & & & & & $\mathrm{Fe} \mathrm{XI}$ & 239.780 & 0.31 \\
\hline \multirow[t]{2}{*}{201.10} & 1.1 & 6.24 & 6.26 & 0.99 & Fe хіII & 201.126 & 0.84 \\
\hline & & & & & $\mathrm{Fe} \mathrm{XI}$ & 201.112 & 0.12 \\
\hline 152.05 & 0.51 & 6.24 & 6.26 & 0.81 & Ni XII & 152.151 & 0.95 \\
\hline 215.16 & 0.21 & 5.42 & 6.29 & 1.47 & $\mathrm{Ov}$ & 215.245 & 0.11 \\
\hline & & & & & S XII & 215.167 & 0.80 \\
\hline 285.80 & 0.23 & 6.27 & 6.30 & 1.49 & S XI & 285.823 & 0.96 \\
\hline 281.40 & 0.32 & 6.28 & 6.30 & 1.05 & S XI & 281.402 & 0.97 \\
\hline 157.75 & 0.30 & 6.30 & 6.31 & 1.33 & Ni XIII & 157.729 & 0.91 \\
\hline 164.13 & 0.24 & 6.31 & 6.32 & 1.01 & Ni XIII & 164.150 & 0.91 \\
\hline 211.40 & 3.9 & 6.29 & 6.32 & 1.03 & Fe XIV & 211.317 & 0.95 \\
\hline 227.50 & 0.30 & 5.43 & 6.33 & 1.62 & S XII & 227.490 & 0.83 \\
\hline 288.35 & 0.35 & 6.35 & 6.36 & 2.29 & S XII & 288.434 & 0.98 \\
\hline 233.90 & 0.42 & 6.34 & 6.37 & 0.61 & $\mathrm{Fe} x \mathrm{v}$ & 233.866 & 0.88 \\
\hline 256.70 & 1.1 & 6.42 & 6.41 & 2.01 & S XIII & 256.685 & 0.97 \\
\hline 263.05 & 0.73 & 6.43 & 6.41 & 0.94 & FexvI & 262.976 & 0.94 \\
\hline 249.17 & 0.40 & 5.64 & 6.43 & 1.20 & Ni xvII & 249.189 & 0.91 \\
\hline 292.00 & 0.21 & 6.56 & 6.46 & 0.85 & Ni xvIII & 291.984 & 0.86 \\
\hline & & & & & Fe xIV & 292.064 & 0.10 \\
\hline 93.93 & 0.016 & 6.86 & 6.48 & 0.78 & Fe xVIII & 93.932 & 0.95 \\
\hline
\end{tabular}


PEVE irradiances with those obtained by EVE in 2010 shows a clear change in all the lines formed above $1 \mathrm{MK}$, which means that the only complete EUV spectrum of the quiet Sun available to date is the spectrum obtained in 2008 , with the few corrections we have noted.

The modelled spectra show a satisfactory level of completeness in the CHIANTI atomic data for these medium-resolution spectra. This is very important for modelling purposes.

The good agreement between predicted and observed quietSun line irradiances was obtained using the zero-density CHIANTI v.8.07 ion abundances. Problems with earlier ion charge states for some iron ions were found and fixed. We experimented with the density-dependent ion abundances calculated with the OPEN-ADAS rates, but found several discrepancies, some by factors of 2 . The only improvement in using the ADAS rates was for a few low-temperature ions. This clearly indicates the need for improved models of the ion charge states, now that excitation data within an ion are relatively accurate. Work on this issue is in progress.

The atomic data for $\mathrm{O}_{\text {II }}$ are clearly incorrect, although a detailed assessment of such low-temperature lines needs to also take into account opacity effects in the lines, which is beyond the scope of this paper.

Contrary to earlier findings (see the reviews in Del Zanna et al. 2002; Del Zanna \& Mason 2018), good agreement between predicted and observed irradiances is found for most anomalous ions of the Li- and Na-like sequences in the upper transition region and corona. Large discrepancies are still present for $\mathrm{O}$ vi, some low-temperature ions, and the helium lines. We clearly need improved models to explain the intensities of these lines, as shown for the helium lines by Golding et al. (2017).

We confirm our previous findings that the quiet solar corona has photospheric abundances. We find excellent agreement in the relative abundances of some high-FIP $(\mathrm{O}, \mathrm{Ne}, \mathrm{S})$ and low-FIP (Fe, $\mathrm{Mg}, \mathrm{Si}, \mathrm{Ca}$ ) elements using the compilation of photospheric values of Asplund et al. (2009). We note that the Ne abundance suggested by Asplund et al. (2009) was not obtained by direct photospheric measurements. The measurements are sound. They involve strong lines emitted by the whole solar disc.

The issue of coronal abundances has recently received renewed interest in the solar community. Several studies based on Hinode EIS have been published (see the review in Del Zanna $\&$ Mason 2018). Some of the published results are puzzling, however. For example, several studies (e.g. Brooks et al. 2015 and references therein) used the $\mathrm{S} \times 264.2 \AA$ line to measure the sulfur abundance relative to that of low-FIP elements (using Six 272. $\AA$ and lines from iron ions). Sulfur has an FIP of about $10 \mathrm{eV}$, but shows abundance variations similar to those of the high-FIP elements in remote-sensing observations, so that it is often used as a proxy to measure the FIP bias. We note that $\mathrm{S} x$ in the quiet Sun is formed around 1.3 MK (cf. Table 1). The results of Brooks et al. (2015) are that the quiet Sun shows an FIP bias of about 2, while active regions have an FIP bias of about 4 . The quiet-Sun results contradict the present results, where the same Sx $264.2 \AA$ line is well represented (within 20\%) with the Asplund et al. (2009) photospheric abundances, as shown in Table 1. We obtain the same results using the Malinovsky \& Heroux (1973) spectrum.

The active region results of Brooks et al. (2015) contradict those obtained for the 1-2 MK diffuse emission in active regions by (Del Zanna 2012a), where an FIP bias of about 2 was found. Clearly, when active regions are present, they produce all the additional plasma above $1 \mathrm{MK}$ that is not present in the quiet Sun, as we have discussed in detail in Paper II and Paper III. The reanalysis of the active Sun observed by Malinovsky \& Heroux (1973) is consistent with the quiet solar corona having photospheric abundances, while the $2 \mathrm{MK}$ plasma clearly has an FIP bias of about 2 .

Acknowledgements. Support by STFC (UK) via the consolidated grant of the DAMTP atomic astrophysics group (ST/P000665/1) at the University of Cambridge is acknowledged. The calculation of the APAP-Network atomic data used in this analysis was funded by STFC via a grant to the University of Strathclyde. CHIANTI is a collaborative project involving George Mason University, the University of Michigan (USA), and the University of Cambridge (UK). The irradiance data used here are courtesy of the NASA/SDO EVE science team. We acknowledge the use of the OPEN-ADAS database, maintained by the University of Strathclyde.

\section{References}

Andretta, V., \& Del Zanna, G. 2014, A\&A, 563, A26

Andretta, V., Del Zanna, G., \& Jordan, S. D. 2003, A\&A, 400, 737

Asplund, M., Grevesse, N., Sauval, A. J., \& Scott, P. 2009, ARA\&A, 47, 481

Badnell, N. R. 2006, ApJS, 167, 334

Badnell, N. R., O’Mullane, M. G., Summers, H. P., et al. 2003, A\&A, 406, 1151

Badnell, N. R., Del Zanna, G., Fernández-Menchero, L., et al. 2016, J. Phys. B At. Mol. Phys., 49, 094001

Bradshaw, S. J., Del Zanna, G., \& Mason, H. E. 2004, A\&A, 425, 287

Brekke, P., Thompson, W. T., Woods, T. N., \& Eparvier, F. G. 2000, ApJ, 536, 959

Brooks, D. H., Ugarte-Urra, I., \& Warren, H. P. 2015, Nat. Commun., 6, 5947 Burgess, A., \& Summers, H. P. 1969, ApJ, 157, 1007

Burton, W. M., Jordan, C., Ridgeley, A., \& Wilson, R. 1971, R. Soc. London Philos. Trans. Ser. A, 270, 81

Chamberlin, P. C., Hock, R. A., Crotser, D. A., et al. 2007, SPIE Conf. Ser., 6689 Chamberlin, P. C., Woods, T. N., Crotser, D. A., et al. 2009, Geophys. Res. Lett., 36,5102

Culhane, J. L., Harra, L. K., James, A. M., et al. 2007, Sol. Phys., 60

Del Zanna, G. 1999, PhD thesis, Univ. of Central Lancashire, UK

Del Zanna, G. 2009a, A\&A, 508, 513

Del Zanna, G. 2009b, A\&A, 508, 501

Del Zanna, G. 2012a, A\&A, 537, A38

Del Zanna, G. 2012b, A\&A, 546, A97

Del Zanna, G. 2013a, A\&A, 555, A47

Del Zanna, G. 2013b, A\&A, 558, A73

Del Zanna, G., \& Andretta, V. 2011, A\&A, 528, A139

Del Zanna, G., \& Andretta, V. 2015, A\&A, 584, A29

Del Zanna, G., \& Badnell, N. R. 2014, A\&A, 570, A56

Del Zanna, G., \& Badnell, N. R. 2016a, MNRAS, 456, 3720

Del Zanna, G., \& Badnell, N. R. 2016b, A\&A, 585, A118

Del Zanna, G., \& DeLuca, E. E. 2018, ApJ, 852, 52

Del Zanna, G., \& Mason, H. E. 2014, A\&A, 565, A14

Del Zanna, G., \& Mason, H. E. 2018, Liv. Rev. Sol. Phys., 15

Del Zanna, G., \& Storey, P. J. 2012, A\&A, 543, A144

Del Zanna, G., \& Storey, P. J. 2013, A\&A, 549, A42

Del Zanna, G., Bromage, B. J. I., Landi, E., \& Landini, M. 2001, A\&A, 379, 708

Del Zanna, G., Landini, M., \& Mason, H. E. 2002, A\&A, 385, 968

Del Zanna, G., Berrington, K. A., \& Mason, H. E. 2004, A\&A, 422, 731

Del Zanna, G., Andretta, V., Chamberlin, P. C., Woods, T. N., \& Thompson, W. T. 2010, A\&A, 518, A49

Del Zanna, G., O’Dwyer, B., \& Mason, H. E. 2011, A\&A, 535, A46

Del Zanna, G., Storey, P. J., Badnell, N. R., \& Mason, H. E. 2012a, A\&A, 541, A90

Del Zanna, G., Storey, P. J., Badnell, N. R., \& Mason, H. E. 2012b, A\&A, 543, A139

Del Zanna, G., Storey, P. J., Badnell, N. R., \& Mason, H. E. 2014, A\&A, 565, A77

Del Zanna, G., Dere, K. P., Young, P. R., Landi, E., \& Mason, H. E. 2015a, A\&A, 582, A56

Del Zanna, G., Andretta, V., Wieman, S., \& Didkovsky, L. 2015b, A\&A, 581, A25

Del Zanna, G., Badnell, N. R., Fernández-Menchero, L., et al. 2015c, MNRAS, 454, 2909

Del Zanna, G., Raymond, J., Andretta, V., Telloni, D., \& Golub, L. 2018, ApJ, 865,132

Dere, K. P. 2007, A\&A, 466, 771

Dere, K. P., Landi, E., Mason, H. E., Monsignori Fossi, B. C., \& Young, P. R. 1997, A\&AS, 125, 149

Dere, K. P., Landi, E., Young, P. R., et al. 2009, A\&A, 498, 915 
Dere, K. P., Del Zanna, G., Young, P. R., Landi, E., \& Sutherland, R. 2019, ApJs, 241,22

Doschek, E. E., Laming, J. M., Doschek, G. A., Feldman, U., \& Wilhelm, K 1999, ApJ, 518, 909

Dudík, J., Del Zanna, G., Dzifčáková, E., Mason, H. E., \& Golub, L. 2014, ApJ, 780, L12

Dufresne, R. P., \& Del Zanna, G. 2018, A\&A, submitted

Fernández-Menchero, L., Del Zanna, G., \& Badnell, N. R. 2014a, A\&A, 566, A104

Fernández-Menchero, L., Del Zanna, G., \& Badnell, N. R. 2014b, A\&A, 572, A115

Fontenla, J. M., Avrett, E. H., \& Loeser, R. 1993, ApJ, 406, 319

Golding, T. P., Leenaarts, J., \& Carlsson, M. 2017, A\&A, 597, A102

Harrison, R. A., Sawyer, E. C., Carter, M. K., et al. 1995, Sol. Phys., 162, 233

Heroux, L., Cohen, M., \& Higgins, J. E. 1974, J. Geophys. Res., 79, 5237

Hock, R., Woods, T., Eparvier, F., \& Chamberlin, P. 2010, in 38th COSPAR Scientific Assembly, COSPAR Meeting, 38, 5
Laming, J. M. 2015, Liv. Rev. Sol. Phys., 12

Laming, J. M., Drake, J. J., \& Widing, K. G. 1995, ApJ, 443, 416

Landi, E., \& Young, P. R. 2009, ApJ, 706, 1

Landi, E., Feldman, U., \& Dere, K. P. 2002a, ApJ, 574, 495

Landi, E., Feldman, U., \& Dere, K. P. 2002b, ApJS, 139, 281

Landi, E., Young, P. R., Dere, K. P., Del Zanna, G., \& Mason, H. E. 2013, ApJ, 763,86

Malinovsky, L., \& Heroux, M. 1973, ApJ, 181, 1009

Thomas, R. J., \& Neupert, W. M. 1994, ApJS, 91, 461

Wang, T., Thomas, R. J., Brosius, J. W., et al. 2011, ApJS, 197, 32

Wilhelm, K., Curdt, W., Marsch, E., et al. 1995, Sol. Phys., 162, 189

Woods, T. N., Eparvier, F. G., Bailey, S. M., et al. 2005, J. Geophys. Res. (Space Physics), 110, 1312

Woods, T. N., Chamberlin, P. C., Harder, J. W., et al. 2009, Geophys. Res. Lett., 36,1101

Woods, T. N., Eparvier, F. G., Hock, R., et al. 2012, Sol. Phys., 275, 115

Young, P. R., Landi, E., \& Thomas, R. J. 1998, A\&A, 329, 291

\section{Appendix A: Line list}

Table A.1 presents the full list of observed and predicted EUV irradiances for the main, stronger lines in the 60-1040 A range.

Table A.1. EUV irradiances of the quiet Sun.

\begin{tabular}{|c|c|c|c|c|c|c|c|}
\hline$\lambda_{\mathrm{obs}}(\AA)$ & $I_{\mathrm{obs}}$ & $\log T_{\max }$ & $\log T_{\text {eff }}$ & $R$ & Ion & $\lambda_{\exp }(\AA)$ & ratio \\
\hline \multirow[t]{4}{*}{86.91} & 0.18 & 5.93 & 6.04 & 0.39 & Mg VIII & 87.022 & 0.14 \\
\hline & & & & & Mg VIII & 86.844 & 0.21 \\
\hline & & & & & Si VII & 86.914 & 0.13 \\
\hline & & & & & Fe XI & 86.772 & 0.31 \\
\hline \multirow{2}{*}{88.13} & 0.07 & 5.84 & 6.03 & 0.77 & $\mathrm{Ne}$ VIII & 88.120 & 0.25 \\
\hline & & & & & Ne VIII & 88.092 & 0.49 \\
\hline \multirow[t]{2}{*}{88.98} & 0.08 & 6.15 & 6.11 & 0.90 & $\mathrm{Fe} \mathrm{XI}$ & 89.178 & 0.19 \\
\hline & & & & & Fe XI & 88.933 & 0.63 \\
\hline 94.14 & 0.12 & 6.04 & 6.05 & 0.40 & Fe $x$ & 94.012 & 0.74 \\
\hline \multirow[t]{5}{*}{95.42} & 0.10 & 5.74 & 5.95 & 0.54 & Mg VII & 95.423 & 0.12 \\
\hline & & & & & Mg VI & 95.483 & 0.12 \\
\hline & & & & & Si VI & 95.555 & 0.12 \\
\hline & & & & & $\mathrm{Fe} x$ & 95.339 & 0.10 \\
\hline & & & & & $\mathrm{Fex}$ & 95.374 & 0.20 \\
\hline \multirow[t]{2}{*}{96.10} & 0.16 & 6.06 & 6.05 & 0.75 & $\mathrm{Fe} x$ & 96.121 & 0.31 \\
\hline & & & & & Fex & 96.007 & 0.59 \\
\hline \multirow[t]{4}{*}{98.12} & 0.22 & 5.80 & 6.01 & 0.54 & Ne VIII & 98.116 & 0.20 \\
\hline & & & & & $\mathrm{Ne}$ VIII & 98.261 & 0.35 \\
\hline & & & & & $\mathrm{Ne}$ VII & 97.502 & 0.11 \\
\hline & & & & & $\mathrm{Fex}$ & 97.838 & 0.14 \\
\hline \multirow[t]{2}{*}{100.67} & 0.10 & 5.70 & 6.04 & 0.54 & Si vi & 100.953 & 0.13 \\
\hline & & & & & Fe XI & 100.575 & 0.59 \\
\hline \multirow[t]{2}{*}{103.40} & 0.16 & 5.91 & 6.02 & 0.86 & $\mathrm{Ne}$ VIII & 103.085 & 0.19 \\
\hline & & & & & Fe IX & 103.566 & 0.67 \\
\hline 105.20 & 0.12 & 5.91 & 6.01 & 0.48 & Fe IX & 105.208 & 0.87 \\
\hline \multirow[t]{3}{*}{111.59} & 0.17 & 5.72 & 5.95 & 0.34 & Mg VII & 111.984 & 0.12 \\
\hline & & & & & Fe IX & 111.791 & 0.11 \\
\hline & & & & & Fe IX & 112.096 & 0.19 \\
\hline \multirow[t]{3}{*}{113.75} & 0.10 & 5.93 & 6.01 & 1.12 & Fe IX & 113.793 & 0.46 \\
\hline & & & & & Fe IX & 114.024 & 0.24 \\
\hline & & & & & Fe IX & 114.111 & 0.11 \\
\hline \multirow[t]{3}{*}{116.87} & 0.13 & 5.75 & 5.99 & 0.66 & Ne VII & 116.691 & 0.24 \\
\hline & & & & & Fe IX & 116.803 & 0.14 \\
\hline & & & & & Fe IX & 116.803 & 0.15 \\
\hline \multirow[t]{2}{*}{131.12} & 0.11 & 5.74 & 5.93 & 0.96 & Fe VIII & 130.941 & 0.37 \\
\hline & & & & & Fe VIII & 131.240 & 0.56 \\
\hline
\end{tabular}

Notes. Same layout as in Table 1 . The full table is available only at CDS. 